\title{
List Mode
}

National Cancer Institute

\section{Source}

National Cancer Institute. List Mode. NCI Thesaurus. Code C94923.

A data acquisition approach in which detector counts are stored sequentially as scan time progresses. This contrasts with the much more compact storage of time-averaged data in defined frames having pre-selected times and durations. 